\title{
Activity-induced radial velocity jitter in a flaring $M$ dwarf ${ }^{\star}$
}

\author{
A. Reiners ${ }^{\star \star}$ \\ Universität Göttingen, Institut für Astrophysik, Friedrich-Hund-Platz 1, 37077 Göttingen, Germany \\ e-mail: Ansgar.Reiners@phys.uni-goettingen.de
}

Received 26 May 2008 / Accepted 3 March 2009

\begin{abstract}
We investigate the effect of stellar activity and flares on short-term radial velocity measurements in the mid-M flare star CN Leo. Radial velocity variations are calculated from 181 UVES spectra obtained during three nights. We searched for spectral orders that contain very few atmospheric absorption lines and calibrated them against the telluric $A$-band from $\mathrm{O}_{2}$ in the Earth's atmosphere. One giant flare occurred during our observations, which has a very strong effect on radial velocity. The apparent radial velocity shift due to the flare is several hundred $\mathrm{m} \mathrm{s}^{-1}$ and clearly correlated with $\mathrm{H} \alpha$ emission. Outside the flare, only spectral orders containing the most prominent emission lines of $\mathrm{H}, \mathrm{He}$, and $\mathrm{Ca}$ show a correlation to chromospheric activity together with a radial velocity jitter exceeding a few $10 \mathrm{~m} \mathrm{~s}^{-1}$. We identify a number of spectral orders that are free of strong emission lines and show no flaring-related radial velocity jitter, although flares occurred as strong as 0.4 dex in normalized $\mathrm{H} \alpha$ luminosity. The mean radial velocity jitter due to moderate flaring is less than $10 \mathrm{~m} \mathrm{~s}^{-1}$. Strong flares are easily recognized directly in the spectra and should be neglected for planet searches.
\end{abstract}

Key words. stars: activity - stars: late-type - stars: individual: CN Leo - instrumentation: spectrographs - techniques: radial velocities

\section{Introduction}

Radial velocity searches have led to a great many detections of extrasolar planets. Most of these planets are discovered around stars of spectral types $\mathrm{F}, \mathrm{G}$, and $\mathrm{K}$, although radial velocity amplitudes are greater in stars of later spectral type and lower mass. The two reasons for the lack of planet detections around $\mathrm{M}$ dwarfs are a) the intrinsic faintness of $\mathrm{M}$ dwarfs render the detection of radial velocity variations very difficult; and b) many $\mathrm{M}$ dwarfs are very active, which is thought to be a problem for radial velocity detections, and it is sometimes thought to make $\mathrm{M}$ dwarfs less attractive in the quest for habitable planets.

At the time of writing, 13 planets have been detected around $9 \mathrm{M} \mathrm{dwarfs}^{1}$. One of them, Gl 581, is hosting two planets that potentially fall into the star's habitable zone (von Bloh et al. 2007; Selsis et al. 2007). Recently, Tarter et al. (2007) has concluded that $\mathrm{M}$ dwarfs should be included in the quest for habitable worlds and evidence of life, because they are so numerous and there are no obvious reasons why activity should destroy life on them. With the growing importance of $\mathrm{M}$ dwarfs, active or inactive, it is important to understand the systematic difficulties in detecting radial velocity variations.

With the radial velocity technique, one detects planets by measuring radial velocity variations due to the orbital reflex motion of the host star, which is typically on the order of a few $\mathrm{m} \mathrm{s}^{-1}$ depending on stellar and planetary mass. Radial velocities in general are determined by measuring the effective blue- or redshift of a spectrum relative to a reference spectrum. Such a shift

\footnotetext{
* Based on observations collected at the European Southern Observatory, Paranal, Chile, 077.D-0011.

$\star \star$ Emmy Noether Fellow.

1 http://exoplanet.eu
}

in wavelength can either be imposed by a real velocity variation or by a change in the appearance of the star's spectrum. The latter may stem from a cool spot on the surface of a star effectively covering a part of the star's surface. Depending on the surface velocity on a rotating star, a spot can cause virtual radial velocity variations well above the $\mathrm{m} \mathrm{s}^{-1}$ level. Desort et al. (2007) simulated the effect of starspots on the surface of F-, G-, and K-type stars rotating at moderate rotation velocities (up to $v \sin i=7 \mathrm{~km} \mathrm{~s}^{-1}$ ). They find that starspots can lead to virtual radial velocity shifts of several ten $\mathrm{m} \mathrm{s}^{-1}$; a spot covering a fraction of $1.07 \%$ of the surface of a G2 dwarf rotating at $v \sin i=3 \mathrm{~km} \mathrm{~s}^{-1}$ causes a radial velocity variation with an amplitude of $60 \mathrm{~m} \mathrm{~s}^{-1}$.

The main effect of cool starspots is to remove (or diminish) the flux coming from the part that is covered with a spot. Cool spots on the Sun are regions of strong magnetic fields that are several hundred to a thousand $\mathrm{K}$ cooler than their surroundings. The higher atmospheric layers are heated by magnetic energy so that in the vicinity of cool spots chromospheric lines like $\mathrm{H} \alpha$ become visible in emission. In very cool stars like $\mathrm{M}$ dwarfs, not much is known about the properties of starspots and their effect on radial velocity measurements. Bonfils et al. (2007) and Demory et al. (2007) report radial velocity variations due to starspots around the planet hosting M dwarfs Gl 674 and Gl 436. The rotational periods are $35 \mathrm{~d}$ and $48 \mathrm{~d}$, respectively, and semiamplitudes $K_{\mathrm{s}} \approx 5 \mathrm{~m} \mathrm{~s}^{-1}$ were measured. This is roughly consistent with estimates of $K_{\mathrm{s}}$ from Saar \& Donahue (1997), although their estimates are for a G2 dwarf with spot temperature zero, which certainly differs significantly from the situation in M dwarfs.

In very cool stars, the contrast between chromospheric emission lines and the quiet surface is much higher than in hotter stars 


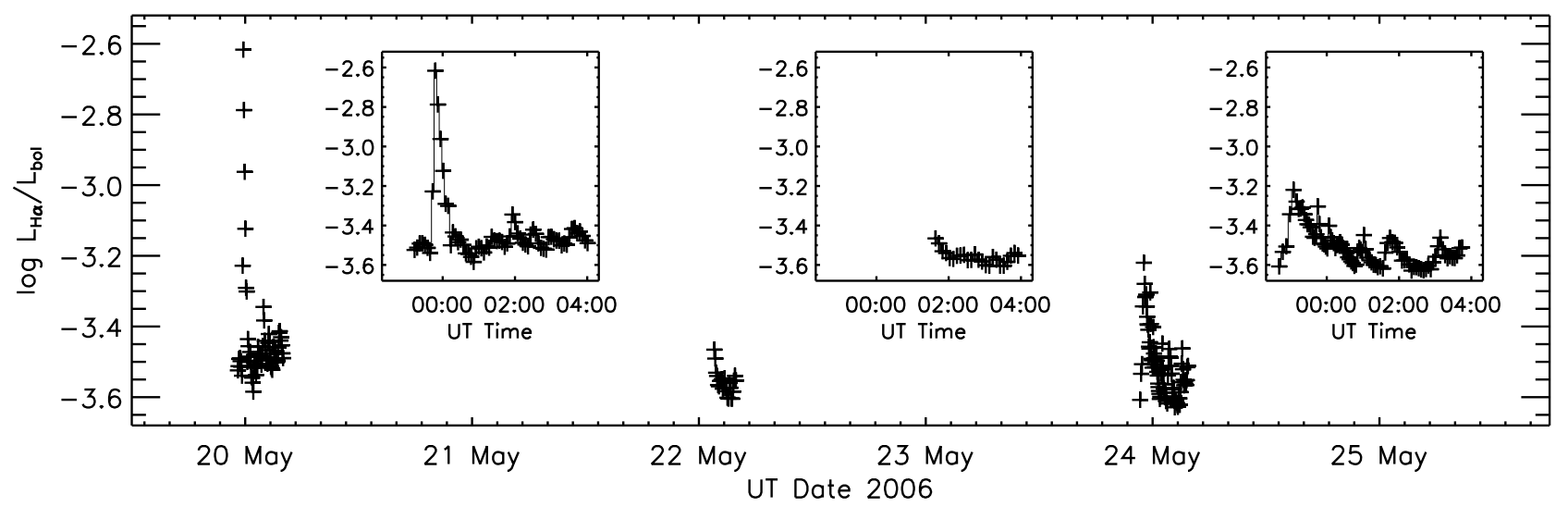

Fig. 1. Normalized $\mathrm{H} \alpha$ luminosity during the three nights of observation. Time coverage of the three half-nights is shown in the plot. The three nights are shown individually in the insets, where the variability in $\mathrm{H} \alpha$ can be seen. A huge flare is clearly visible at the beginning of the first night.

so that observation of activity is easier in cooler stars. A forest of emission lines becomes visible during strong flares in $\mathrm{M}$ dwarfs (see e.g. Fuhrmeister et al. 2008). The influence of active regions on the spectra of M dwarfs, however, has not been studied in a systematic way. One part of the problem is that simulating chromospheric emission is much more difficult than simulating cool starspots. Nevertheless, it is expected that strong activity like flares have strong impact on the radial velocity measurements in M dwarfs.

Kürster et al. (2003) measured radial velocity variations in the inactive M dwarf Gl 699 (Barnard's star). They found an anti-correlation between radial velocity and the strength of the $\mathrm{H} \alpha$ line. In the spectrum of $\mathrm{Gl} 699, \mathrm{H} \alpha$ is always seen in absorption. Even in the "flare" spectrum that Kürster et al. show, $\mathrm{H} \alpha$ is not seen in emission but the absorption is weaker than in the other spectra (an effect that may actually be due to lower temperature not higher; see, e.g., Short \& Doyle 1998). Kürster et al. found a significant anti-correlation between their $\mathrm{H} \alpha$ activity level and radial velocity. The small activity variations of G1 699 led to radial velocity variations on the order of $10 \mathrm{~m} \mathrm{~s}^{-1}$. However, the radial velocity measured in the "flare" spectrum of Kürster et al. does not show as high a radial velocity shift as could be expected from the correlation seen at lower $\mathrm{H} \alpha$ level.

The fact that cool spots on sun-like stars can cause large radial velocity variations, and the result that the $\mathrm{H} \alpha$ line strength (anti-)correlates with radial velocities even in a very inactive $\mathrm{M}$ dwarf raise the question to what extent activity and flaring events influence the detectability of real radial velocity variations due to orbital motion. This is particularly important since in mid- and late-M dwarfs the fraction of active dwarfs rises dramatically, and the majority of mid- and late-M dwarfs show $\mathrm{H} \alpha$ in emission (West et al. 2004).

\section{Data}

In a multi-wavelength study of the very active nearby M6 dwarf CN Leo (Wolf 359, Gl 406), we obtained high resolution spectra with UVES at the Very Large Telescope, Paranal, Chile. The purpose of this data was to investigate the multi-wavelength behaviour of flares. Here, we investigate this data for radial velocity variations. The target always shows strong $\mathrm{H} \alpha$ emission, and during the three nights it presented many small flares and one very big one.

We obtained 181 spectra during three half-nights in 2006 (May 19/20, 21/22, and 23/24). The data are described in detail in Fuhrmeister et al. (2008) where a spectral atlas of a spectrum taken during a huge flare is presented. The original purpose of the data was to investigate flares, not to search for radial velocity varations. Hence, no special calibrations were done and the spectra were taken without the iodine absorption cell. We used a slit width of $1^{\prime \prime}(R \sim 40000)$ and the red arm was centered at $830 \mathrm{~nm}$ providing wavelength coverage from $640 \mathrm{~nm}$ to $1008 \mathrm{~nm}$ in 32 orders.

Three half-nights were granted to the project. Because of bad weather conditions, no observations were taken during the first half of the second half-night. During the other two nights, CN Leo was observed more than four hours without interruption. Exposure times varied between $100 \mathrm{~s}$ and $300 \mathrm{~s}$ depending on weather conditions. Several flares occurred during our observations, among them a very strong one that boosted the observed $\mathrm{H} \alpha$ emission by more than an order of magnitude (it saturated the detector so we cannot measure the total $\mathrm{H} \alpha$ emission in that spectrum). The flare is easily visible in the whole optical region, it is discussed in detail in Fuhrmeister et al. (2008).

In Fig. 1, normalized $\mathrm{H} \alpha$ activity is shown during the observations. Measured $\mathrm{H} \alpha$ equivalent widths were converted to $L_{\mathrm{H} \alpha} / L_{\text {bol }}$ using theoretical PHOENIX spectra as a reference (see Reiners \& Basri 2007). Figure 1 shows the temporal coverage of the data. In the insets, the three half-nights are shown individually. The strong variability of CN Leo is obvious from this plot, and the huge flare during the beginning of the first night stands out as an extraordinary event.

\section{Radial velocities}

The most successful method detecting extra-solar planets so far is the radial velocity method. The hunt for radial velocity perturbations induced by planetary companions became possible when this technique became sophisticated enough to push the stability of radial velocity measurements down to several ten $\mathrm{m} \mathrm{s}^{-1}$, i.e. typically only $1 / 100$ of a pixel and less. To reach such an accuracy, wavelength calibration data is included in the target spectrum itself, so that any instrumental effects are directly visible during the observation. One way to reach this is to obtain the spectrum of a calibration lamp next to the target spectrum (the "ThAr method", see Pepe et al. 2002). A second method uses an absorption cell in front of the spectrograph. Light from the star passes the cell, which imprints a dense forest of absortion lines onto the spectrum (Butler et al. 1996). The crucial advantage of both methods is that every systematic effect in the wavelength solution of the spectrum is directly monitored during observation (although the ThAr method requires much higher stability). 


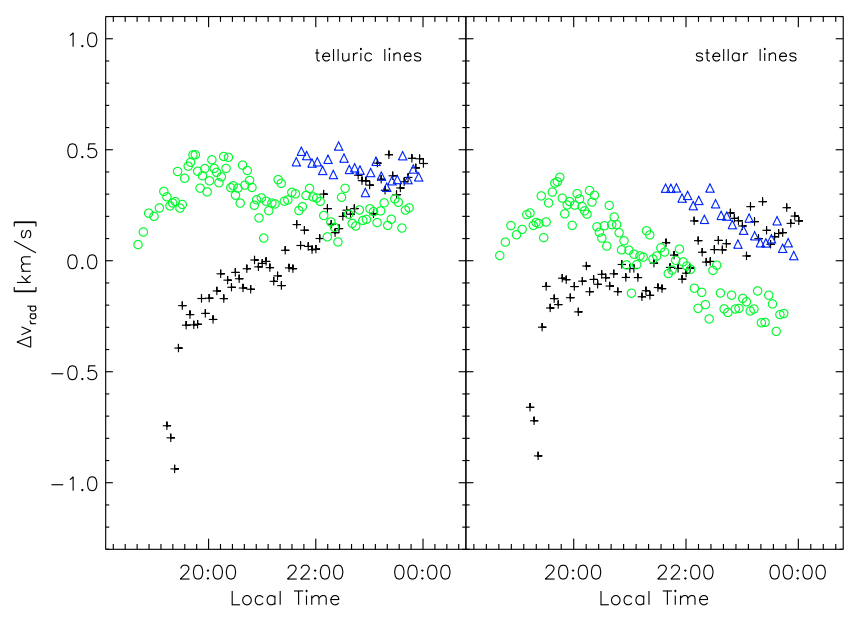

Fig. 2. Relative radial velocities $\Delta v_{\text {rad }}$ between the 181 spectra and an arbitrary reference frame (\#24) versus local time at Paranal. Black crosses are data from the first night, blue triangles are from the second night, and green circles are spectra taken during night 3. Left: radial velocities calculated in the telluric A-band; right: $\Delta v_{\text {rad }}$ calculated from order \#69 that contains mainly the TiO IR band (see text).

Our spectra were not taken with special care regarding to wavelength stability. For calibration of UVES spectra, a ThAr spectrum is usually taken at the end of every night. These are the wavelength references that we use to calibrate our data. The typical stability of UVES, however, is on the order of a pixel over the course of a night, i.e. deviations on the order of a $\mathrm{km} \mathrm{s}^{-1}$ can be expected. In order to reach an accuracy better than the absolute stability of the UVES spectrograph, we employ the absorption lines from Earth's atmosphere. They provide a reliable reference frame on the order of the wind speed, which does not vary abruptly during a night (Balthasar et al. 1982). None of the telluric lines were removed from the spectra prior to analysis.

\subsection{Relative radial velocity shifts}

To measure radial velocity shifts from our spectra, we first calculated the cross correlation functions relative to a reference spectrum. Spectrum number 24 obtained during the first night was used as reference spectrum; it exhibits relatively little $\mathrm{H} \alpha$ emission. This was done for all spectral orders individually, i.e. we calculated $181 \times 31$ individual radial velocity differences. In the following, these are labelled the relative radial velocity shifts (relative to an arbitrary zero point). They are not corrected for instrumental effects that may have occurred during the course of each night.

Figure 2 shows all 181 relative radial velocities $\Delta v_{\text {rad }}$. Black crosses are data taken during the first night, blue triangles are taken during the second night, and green circles are observations from the third night. In the left panel, $\Delta v_{\text {rad }}$ from order \#80 $(757-769 \mathrm{~nm})$ is shown. This order covers the A-band due to atmospheric $\mathrm{O}_{2}$. Assuming no or very little variations in the telluric lines, $\Delta v_{\text {rad }}$ from this order shows the absolute drift of the spectrograph during the nights. In the right panel of Fig. 2, we show radial velocity differences calculated from spectral order \#69 that contains the IR TiO band and only very little telluric absorption. No barycentric correction was applied to the data shown in Fig. 2.

Clearly, relative radial velocities of telluric lines and stellar lines follow the same gross pattern. During the first night (black crosses), the telluric and the stellar spectra show a strong redward shift within the first 15 minutes. More precisely, a blueshift occurs during the first three exposures followed by a steep redward jump. After that, a linear redward trend drifting approximately $1 \mathrm{~km} \mathrm{~s}^{-1}$ in four hours occurred. This trend, observed both in the atmospheric and the stellar lines, clearly is introduced by the UVES instrument itself and cannot be due to the motion of the star. The origin of the jump and the linear redward trend during the first night is unclear, but a thorough analysis of instrumental effects goes beyond the scope of this paper. During the second night (blue triangles) and the third night (green circles), a shallow blueward trend is observed. At the beginning of the third night, a redward shift occurred that is similar to the one observed in the first night. The blueward drift during the second and third nights is on the order expected from changes due to variable atmospheric pressure inside the instrument (see next section).

\subsection{Differential radial velocity shifts}

Calibrating radial velocity shifts with telluric lines has several caveats that must be taken into account. The basic problem is that telluric lines are spread over almost the entire spectrum, but in particular in the rich spectra of M dwarfs they are not always easily discovered. The cross-correlation function of two spectra taken from the same star generally consists of two components: 1 . the correlation peak from stellar features, which follow the motion of the star and the drift due to barycentric motion. 2. The correlation peak of the telluric lines that are not subject to barycentric velocity drifts. If a spectral range contains both sets of features, the influence of the correlation peak due to telluric lines will affect the peak from stellar features. In the easiest case, telluric features dominate and the correlation peak no longer follows the star's motion but rather the effects of telluric lines. In a more complicated situation, the varying distance between the stellar and the telluric peaks interfere so that the velocity measured from the cross correlation function is a complex combination of stellar and barycentric motion.

One way to get rid of telluric features is to correct for them by using either a telluric standard spectrum or a synthetic set of telluric lines. Alternatively, one can mask out regions of telluric lines and use only regions that are virtually free of telluric contamination. As mentioned above, it is not always straightforward to identify telluric features in particular in the spectra of M dwarfs. Reiners et al. (2007) show a high resolution spectrum of a standard star in which telluric absorption at Paranal is visible in the wavelength region used for this work. This spectrum shows that telluric contamination is relatively weak outside the strong bands of $\mathrm{O}_{2}(A$ - and $B$-band at $760 \mathrm{~nm}$ and $690 \mathrm{~nm}$, respectively) and the $\mathrm{H}_{2} \mathrm{O}$ features at $720 \mathrm{~nm}, 820 \mathrm{~nm}$, and between $890 \mathrm{~nm}$ and $980 \mathrm{~nm}$. This means that probably a large range of the spectral coverage of UVES can be used to measure the radial velocity shift of CN Leo.

In this work, we chose neither to remove nor to mask telluric features. Instead, we computed the cross correlation function for each order individually and search for orders that trace the barycentric velocities. We assume that the velocities calculated from these orders follow the apparent radial velocity of the target.

With the relative radial velocity shift with respect to one reference frame, we can now calculate the differential radial velocity shift $\Delta v_{\text {rad,star }}-\Delta v_{\text {rad,telluric }}=\Delta v_{\mathrm{s}}-\Delta v_{\mathrm{t}}$, i.e. the residual between the relative telluric shift from order \#80 (left panel of Fig. 2) and the relative stellar shift (one order shown in the right panel of Fig. 2). The differential radial velocities for all orders 

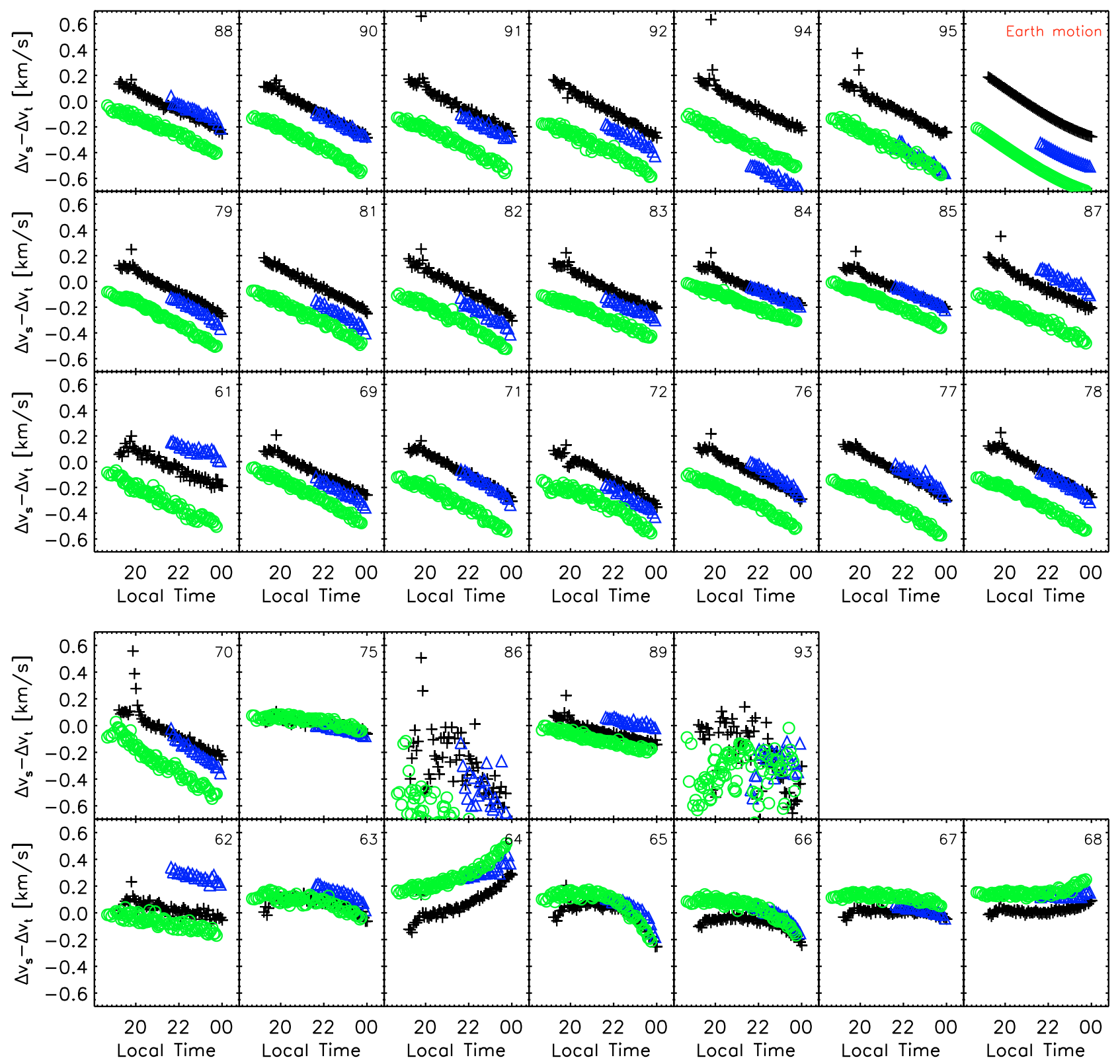

Fig. 3. Differential radial velocities without barycentric corrections for all spectral orders individually. Black crosses: first night; blue triangles: second night; green circles: third night. Top three panels show orders that resemble the pattern of barycentric motion. These orders are compiled in Table 1. The barycentric motion is shown in the upper right plot. Lower two panels show spectral orders not following barycentric motion. These orders are affected by telluric lines or other contaminants, they are compiled in Table 2.

are shown in Fig. 3. We group the orders into "stellar" and "telluric" orders based on whether or not they trace the barycentric motion. The three top panels show "stellar" orders following the apparent velocity introduced by the motion of Earth, which itself is shown in the upper right panel of Fig. 3. These orders are compiled with their wavelength coverage in Table 1 . The amplitude of the drift introduced by the motion of Earth is on the order of $500 \mathrm{~m} \mathrm{~s}^{-1}$ per $4 \mathrm{~h}$ and clearly dominates the radial velocity signal measured during each night in the 20 orders shown here. The radial velocity differences between the three individual nights (sets of black crosses, blue triangles, and green circles) do not strictly resemble the pattern expected from barycentric motion. This is not suprising because the absolute stability of the spectrograph is not better than a few hundred $\mathrm{m} \mathrm{s}^{-1}$ from night to night. The differences between the first and third night are in general closer to the expectations than the differences between the second and the other two nights. The differences vary between different orders. We calculate the median of the differential radial velocity shifts from the first and second nights and plot their differences in Fig. 4 as a function of spectral order. The offset between spectra taken during the first and the second night varies between $\sim-500$ and $300 \mathrm{~m} \mathrm{~s}^{-1}$ and shows relatively smooth variation with wavelength, which indicates that the offset is introduced by inaccuracies of the dispersion relation. Thus, UVES data calibrated with only one ThAr frame per night can clearly not be used to search for radial velocity variations on the order of $100 \mathrm{~m} \mathrm{~s}^{-1}$ over several hours. On the other hand, short-time radial velocity variations can be traced as will be shown in the following.

The two bottom panels of Fig. 3 show differential radial velocities in the spectral orders that do not follow the drift expected from barycentric motion. These are the orders that are either affected by large scatter caused by broad features not suitable for 
Table 1. Spectral orders of UVES that contain mostly stellar absorption features. Orders with relevant emission lines or telluric absorption lines are excluded.

\begin{tabular}{lc}
\hline \hline Order \# & Wavelength coverage [nm] \\
\hline 61 & $992-1008$ \\
69 & $877-891$ \\
71 & $853-865$ \\
72 & $841-853$ \\
76 & $796-809$ \\
77 & $785-798$ \\
78 & $775-788$ \\
79 & $766-778$ \\
81 & $747-759$ \\
82 & $738-750$ \\
83 & $730-739$ \\
84 & $721-730$ \\
85 & $713-721$ \\
87 & $697-705$ \\
88 & $687-699$ \\
90 & $672-683$ \\
91 & $666-674$ \\
92 & $659-666$ \\
94 & $643-654$ \\
95 & $637-647$ \\
\hline
\end{tabular}

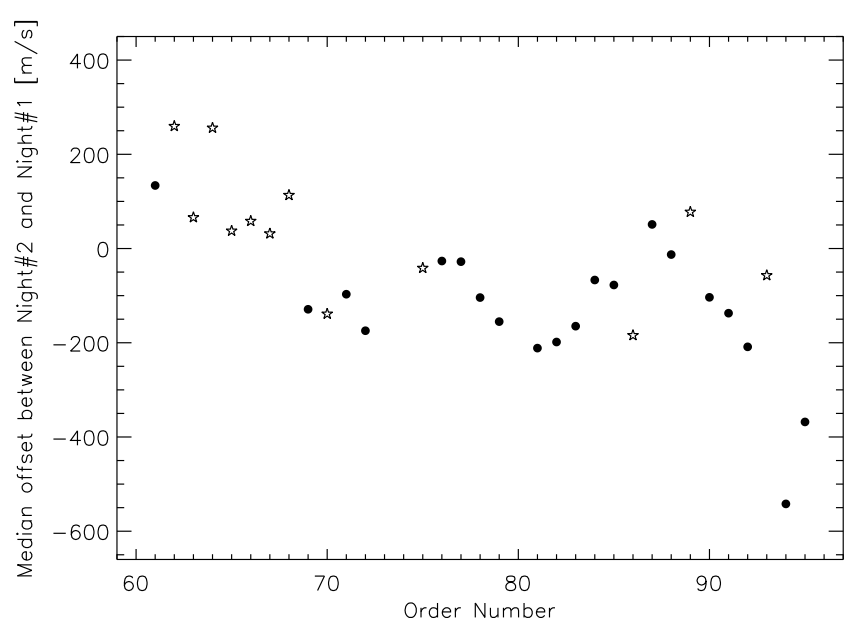

Fig. 4. Offset between the median differential radial velocities during night \#2 and night \#1 as a function of spectral order (difference between blue triangles and black crosses in Fig. 3). Filled circles are from orders tracing the star's motion, open stars show telluric orders (top and bottom panel in Fig. 3), respectively.

precision velocity measurements (orders \#86 and \#93), or that are affected by telluric contamination. Order \#70 was taken out because it follows the weaker flare at the beginning of night 3 (before 20:00), which is probably caused by the Ca emission line. The spectral orders shown in the bottom two panels are not used for radial velocity calculations, they are given in Table 2 together with the source of contamination.

A comparison between the spectral orders of Table 1 and known telluric absorption features shows that some spectral regions, e.g., at $690 \mathrm{~nm}$ and at $720 \mathrm{~nm}$ contain telluric absorption. These orders are likely to be affected by telluric absorption, too, which can be a problem for radial velocity work. At least they should be used only with great care. In our case, Fig. 3 shows that the stellar signal is dominating the radial velocity measurement (e.g., in orders 84 and 88), and we chose to include them in the set of "stellar" orders. Furthermore, a comparison with the telluric standard spectrum in Reiners et al. (2007),
Table 2. Spectral orders of UVES not used for radial velocity calculations. Telluric absorption features or broad stellar features are indicated.

\begin{tabular}{lrr}
\hline \hline Order \# & Wavelength coverage [nm] & feature \\
\hline 62 & $976-991$ & $\mathrm{H}_{2} \mathrm{O}$ \\
63 & $960-976$ & $\mathrm{H}_{2} \mathrm{O}$ \\
64 & $945-960$ & $\mathrm{H}_{2} \mathrm{O}$ \\
65 & $931-945$ & $\mathrm{H}_{2} \mathrm{O}$ \\
66 & $917-931$ & $\mathrm{H}_{2} \mathrm{O}$ \\
67 & $903-917$ & $\mathrm{H}_{2} \mathrm{O}$ \\
68 & $890-903$ & $\mathrm{H}_{2} \mathrm{O}$ \\
70 & $865-877$ & $\mathrm{Ca}$ (stellar) \\
75 & $808-818$ & $\mathrm{H}_{2} \mathrm{O}$ \\
80 & $758-767$ & $\mathrm{O}$ \\
86 & $705-713$ & $\mathrm{He}, \mathrm{TiO}$ (stellar) \\
89 & $681-689$ & $\mathrm{O}$ \\
93 & $652-659$ & $\mathrm{H} \alpha$ (stellar) \\
\hline
\end{tabular}

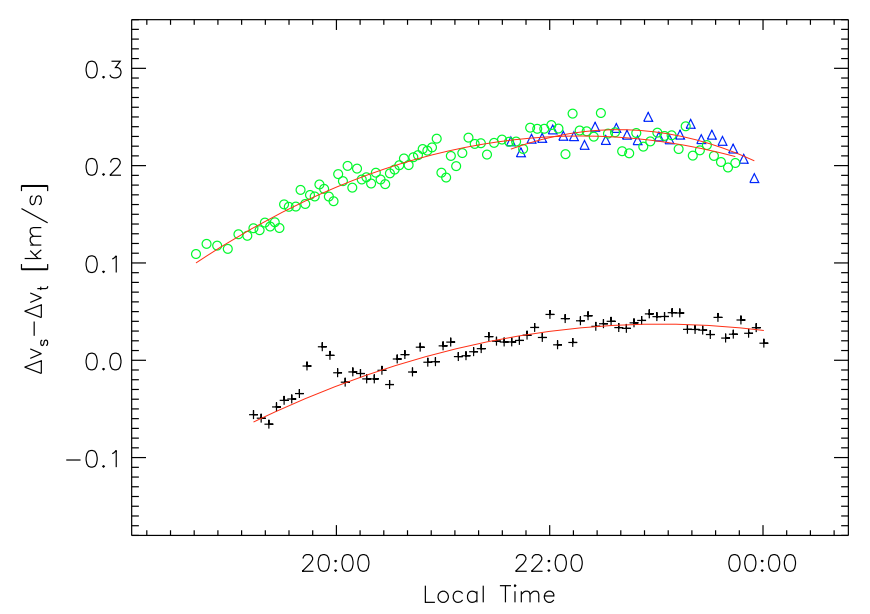

Fig. 5. Differential radial velocities $\Delta v_{\mathrm{s}}-\Delta v_{\mathrm{t}}$ for the three nights versus local time. Symbols are the same as in Fig. 2. A second order polynomial fit for the three nights is overplotted in red. The giant flare at the beginning of the first night was excluded from the fit.

taken with the same instrumental setup, shows that the telluric features identified in Table 2 represent the strong features seen from Paranal. No other significant absorption features (on the order of $20 \%$ depth) appears in this atlas. The relevant parts of the telluric bands at $690 \mathrm{~nm}$ and $720 \mathrm{~nm}$, for example, have less than $15 \%$ depth, which is much weaker than the depth of stellar features. We note that classifying more orders that are suspect to telluric contamination as "telluric" has no significant influence on the results presented in the following.

Our next step is to apply the barycentric correction and to calculate the mean differential radial velocity shift from the "stellar" orders (top three rows of Fig. 3 and Table 1). The mean of the differential velocity shifts from the 20 orders given in Table 1 is shown in Fig. 5.

The differential radial velocity shifts from each of the three nights all lie on smooth curves. Differential velocity shifts from the second and the third night show very similar absolute values. Those from the first night, however, fall about $200 \mathrm{~m} \mathrm{~s}^{-1}$ below the results of the other two nights. This discrepancy is probably connected to the strong drift in the relative radial velocities during the first night. We checked temperatures during the three nights and found that relatively large temperature variations occurred during the first night. We can speculate that this may be connected to the systematic offset between the dispersion relations during that night and the other two nights (Fig. 5). 


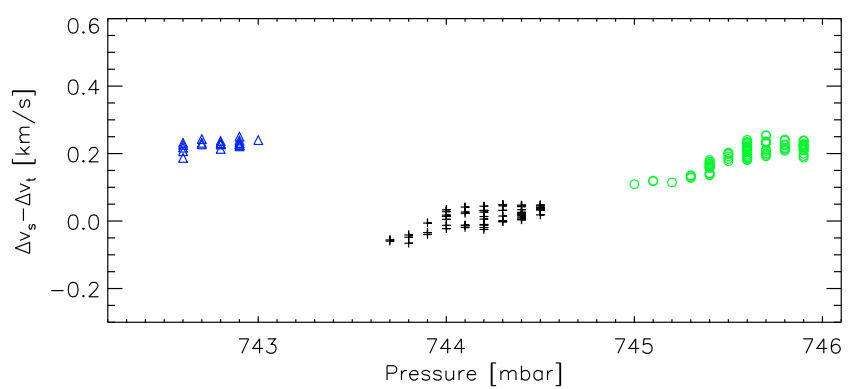

Fig. 6. Differential velocities plotted versus atmospheric pressure during the three nights of observation. Symbols are the same as in Fig. 2.

However, the temperature variations cannot explain the large drift in relative velocities seen in Fig. 2 (the temperature effect is expected to be much smaller, see Kaufer et al. 2007).

The smooth variation of differential radial velocity shifts observed during each of the three nights are all following a very similar pattern. It is not the main objective of this paper to investigate the nature of this smooth pattern, because we are mainly interested in short term variations connected to stellar flares that we can trace in $\mathrm{H} \alpha$. Nevertheless, we can speculate about the reason of the smooth changes seen in each of the three nights. The striking similarity of the nightly variations suggests a reason connected to the nightly course of observations. One possibility is that some interference between telluric lines and stellar lines is left in the orders that we selected above. As explained, this could introduce changes of radial velocity drift with changing barycentric motion. However, the barycentric motion is almost monotonous during each night (upper right box in Fig. 3). The nightly velocity variations consistently show a maximum around 23:00 h each night, which makes a connection to telluric lines improbable (although we cannot completely exclude this option).

A second possibility to explain the intra-night pattern are variations of atmospheric pressure inside the UVES instrument. Pressure variations can alter the dispersion relation and hence affect the differences between wavelength shifts observed at different spectral orders. We checked the behaviour of atmospheric pressure during the three nights; they indeed follow a pattern very similar to the curves in Fig. 5. In Fig. 6, the differential velocity shift is plotted as a function of pressure. For each night, the curves follow a relative well defined line with a similar dependence on pressure while the absolute pressure values are very different. The amplitude of pressure variations is consistent with the expectations from the UVES manual (Kaufer et al. 2007).

\subsection{Could the intra-night drift be real?}

A viable explanation for the smooth trend in differential radial velocities in Fig. 5 is a change of dispersion coupled to atmospheric pressure inside the instrument. However, this explanation cannot be tested, and the trend may as well be real. If we assume that the offset between the differential radial velocities, measured during the first and the other two nights, is instrumental but the drift comes from real radial velocity variations, the "detected" period would be about 2 days or an integer fraction of that. A potential explanation for such a period in the flare star CN Leo are corotating spots producing radial velocity variations with a semi-amplitude of $K_{\mathrm{s}} \sim 100 \mathrm{~m} \mathrm{~s}^{-1}$. As in higher mass stars, spots may produce radial velocity variations on the order of several 10 to $100 \mathrm{~m} \mathrm{~s}^{-1}$ (Bonfils et al. 2007; Demory et al. 2007; Desort et al. 2007). The rotational period of

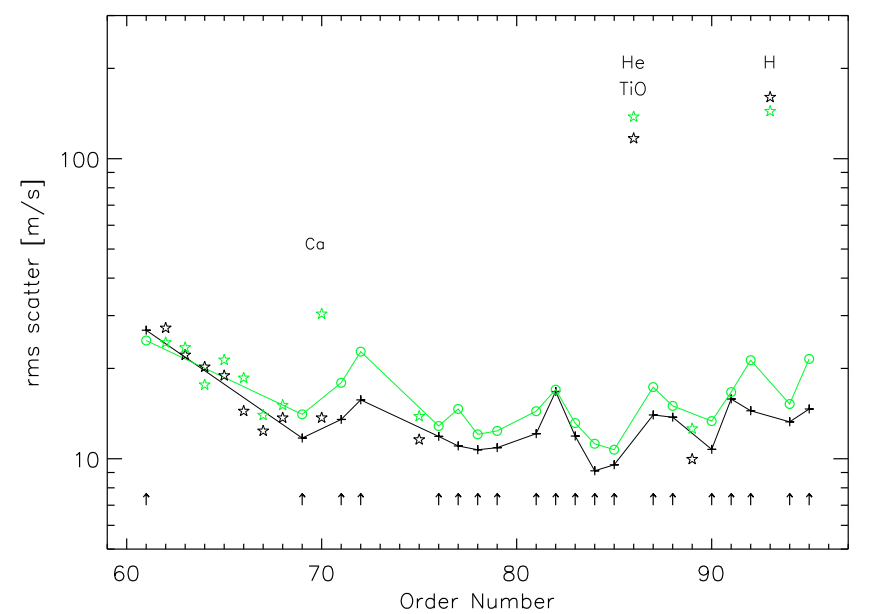

Fig. 7. Scatter of differential radial velocities around the trend fitted in Fig. 5. Only nights 1 (black crosses) and 3 (green circles) are shown. Stars indicate orders that predominantly contain telluric features, arrows mark the orders used to compute stellar radial velocities (these are the orders given in Table 1).

CN Leo is not known, but its surface rotation velocity is about $v \sin i=3 \mathrm{~km} \mathrm{~s}^{-1}$ (Reiners \& Basri 2007). Lacy (1977) gives a value of $\log R / R_{\odot}=-0.84\left(R=0.14 R_{\odot}\right)$ for the radius of $\mathrm{CN}$ Leo. Thus, the (projected) rotational period of CN Leo is $P / \sin i \approx 2 \mathrm{~d}$ ! This means that rotational modulation as a reason for the radial velocity changes seen during each of the three nights is not inconsistent with the expected rotational period of $\mathrm{CN}$ Leo. However, we have tested the periodicity of $\mathrm{H} \alpha$ and other emission lines and found no sign of a rotational period of $2 \mathrm{~d}$. This may imply that the rotational variation is buried under the strong and irregular flaring activity so that CN Leo's rotation cannot be detected in emission lines, but it may also mean that the rotational period of CN Leo is very different from $2 \mathrm{~d}$.

A third way to generate radial velocity variations as observed in our data is an object in orbit around CN Leo. The semiamplitude caused by the hypothetical planet would be around $100 \mathrm{~m} \mathrm{~s}^{-1}$, which could be explained by a planet with a projected mass of $M \sin i \sim 0.2 M_{\mathrm{J}}$ at a distance of $0.015 \mathrm{AU}$. For now, this hypothesis cannot be falsified with the available data, but seems to be extremely unlikely given the close match to pressure changes in the instrument and the coincidence with the estimated rotational period of CN Leo.

\section{Results}

The main focus of this work are the short-term variations of the apparent radial velocity introduced by stellar flares. These flares have timescales of the order of about half an hour.

We removed the intra-night trend from the differential radial velocity shifts in Fig. 5 by applying a second order polynomial fit (exluding the large flare during the first night). The resulting final radial velocities are discussed in the following.

\subsection{Radial velocity jitter}

Figure 7 displays the rms scatter for all UVES orders that are covered by our setup. Again, the six spectra taken during the huge flare were neglected. Only nights 1 and 3 are shown because much less data is available for the second night. Spectral orders affected by telluric lines (Table 2) are marked with a star. 


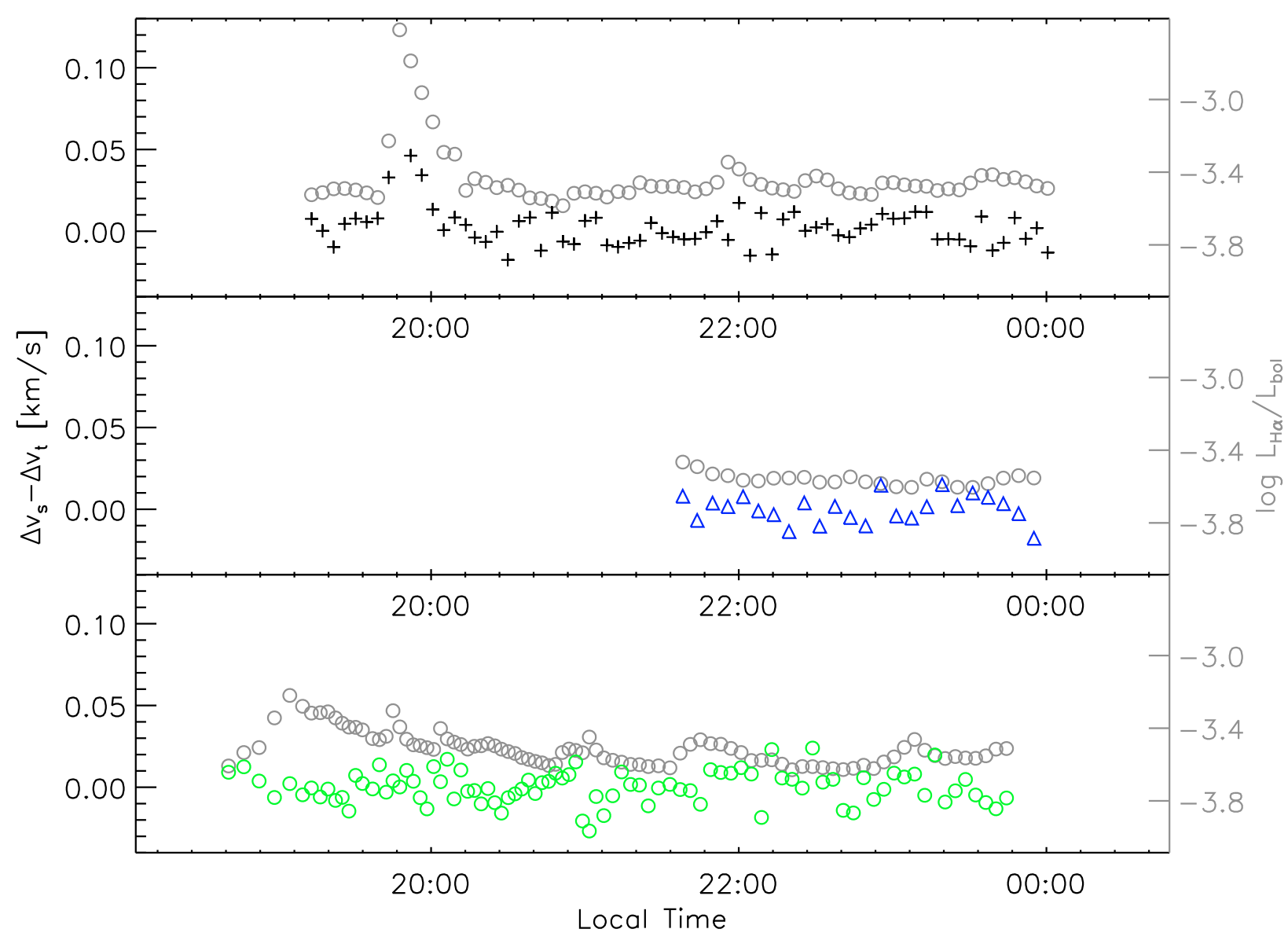

Fig. 8. Radial velocity jitter for the three nights (from top to bottom, symbols as above) together with normalized H $\alpha$ luminosity (grey circles). The polynomials shown in Fig. 5 are subtracted from the differential radial velocities.

Symbols as in the figures above, i.e. black crosses for night 1 and green circles for night 3 , are used for the other orders covering mainly stellar features (Table 1). These orders are connected by black and green lines for the first and third night, respectively. Interestingly, the rms scatter is decreasing among the lowest orders (longest wavelengths) from values around $30 \mathrm{~m} / \mathrm{s}$ in order \#61 down to values around $10 \mathrm{~m} / \mathrm{s}$ in order \#69. The reddest order is virtually free of telluric features but contains many strong stellar lines due to molecular $\mathrm{FeH}$. The next 7 orders predominantly contain absorption due to atmospheric $\mathrm{H}_{2} \mathrm{O}$. The trend of higher scatter in the reddest orders is probably caused by the quality of the wavelength solution, which becomes worse towards the near infrared because the ThAr calibration lamp does not provide as many lines as in the bluer spectral orders.

Most of the orders have rms values between 10 and $20 \mathrm{~m} \mathrm{~s}^{-1}$. Several orders show relatively high rms values. This "scatter" is correlated with $\mathrm{H} \alpha$ emission (see next section) and is caused by chromospheric emission lines contained in these orders. The most prominent lines that affect radial velocity measurements are the lines of the Ca infrared triplet at $849.8 \mathrm{~nm}, 854.2 \mathrm{~nm}$, and $866.2 \mathrm{~nm}$ (orders \#72, \#71, and \#70), two He I lines at $706.5 \mathrm{~nm}$ (order \#86) and $667.8 \mathrm{~nm}$ (orders \#91 and \#92), and $\mathrm{H} \alpha$ (order \#93). Order \#86 also contains the strong TiO $\gamma$-band. This very strong feature is a dense forest of blended lines generating wide correlation functions, which are part of the reason for the relatively large scatter in radial velocity.

In Fig. 8, the mean differential radial velocity shifts calculated from all orders in Table 1 are shown after substracting the smooth trend (Fig. 5). The first night is plotted in the top panel, the second in the middle, and the third night in the bottom panel. In the same figure, the values of normalized $\mathrm{H} \alpha$ emission $\log L_{\mathrm{H} \alpha} / L_{\mathrm{bol}}$ are plotted as grey circles.

\subsubsection{The huge flare}

In Fig. 8, the strong flare that occurred at the beginning of the first night is clearly visible as a spike in radial velocity. Spectra of this huge event are discussed in detail in Fuhrmeister et al. (2008); such a flare is obvious in the spectra of M dwarfs. In the spectrum at flare peak, the effect on radial velocity is as large as several hundred $\mathrm{m} \mathrm{s}^{-1}$ and it shows the opposite $\operatorname{sign}\left(\Delta v_{\mathrm{s}}-\Delta v_{\mathrm{t}}=\right.$ $-660 \mathrm{~m} \mathrm{~s}^{-1}$, not shown in Fig. 8). The exact value of the radial velocity shift during flare peak has nothing to do with the star's radial velocity (it may reflect the geometry and dynamics of the flare). We have not included this point in Fig. 8, but we note that its radial velocity is an order of magnitude larger than in all other spectra. After the flare, the radial velocity shift diminishes together with $\mathrm{H} \alpha$ luminosity.

In a search for radial velocity variations, the spectra affected by a flare as huge as this one are easily distinguished from "quiet" spectra. In a planet search program, they should not be used to detect radial velocity variations.

\subsubsection{Jitter outside the huge flare}

The radial velocities outside the huge flare show no obvious trend or period. The rms scatter for the three nights is $8.2 \mathrm{~m} \mathrm{~s}^{-1}$ for the first night, $8.4 \mathrm{~m} \mathrm{~s}^{-1}$ for the second night, and $9.7 \mathrm{~m} \mathrm{~s}^{-1}$ 


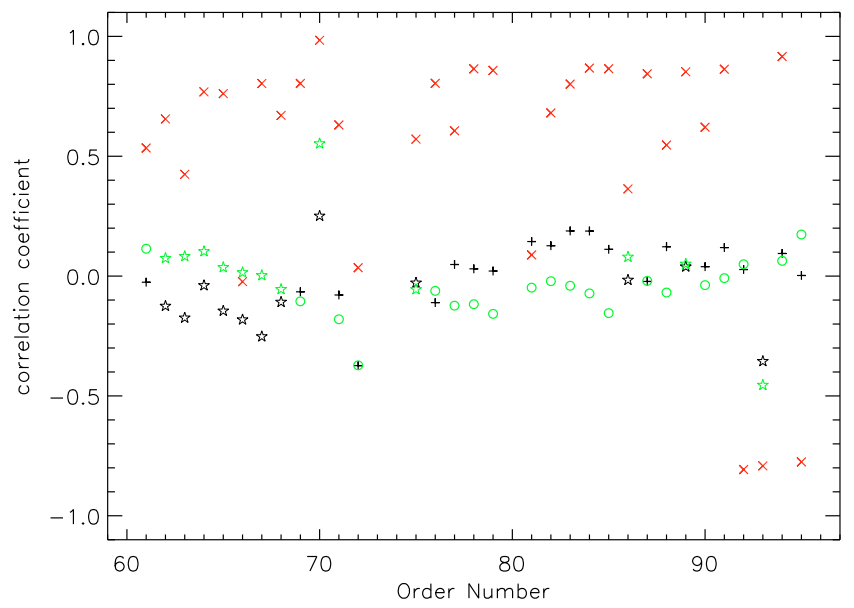

Fig. 9. Correlation coefficients between the radial velocity shifts and normalized $\mathrm{H} \alpha$ luminosities. Black crosses $(+)$ and green circles show correlation coefficients for each order containing mainly stellar features during the first and third nights, respectively (Table 1). Stars show orders of Table 2. As red crosses $(\times)$, the correlation coefficients calculated for the first night are shown including the spectra taken during the huge flare.

for the third night. The overall jitter for all three nights taken together is $9.0 \mathrm{~m} \mathrm{~s}^{-1}$. The meaning of this result is twofold: a) for the purpose of this investigation, the accuracy to which differential radial velocity variations are determined is on the order of $10 \mathrm{~m} \mathrm{~s}^{-1}$; b) outside the giant flare, the still relatively strong flaring activity $\left(\leqslant 0.4\right.$ dex in $\left.\log L_{\mathrm{H} \alpha} / L_{\mathrm{bol}}\right)$ on CN Leo has no effect on radial velocity measurements in excess of $10 \mathrm{~m} \mathrm{~s}^{-1}$ as long as spectral orders are used that avoid strong chromospheric emission lines.

\subsection{Correlation with activity}

In the calculation of the rms jitter above, we excluded the spectra taken during the huge flare. This flare event does show influence on the radial velocity curve, but flares of this strength pose no danger to radial velocity surveys because they are obvious in the spectra; they must not be used for planet searches. However, the data on $\mathrm{H} \alpha$ emission shows that a number of smaller flaring events occurred during the three nights. Are the smaller flares, which are not easily detectable in the spectra and will contribute to radial velocity measurements, affecting the radial velocity measurements? From a first glance, the curves of radial velocity and $\mathrm{H} \alpha$ luminosity in Fig. 8 show no striking similarity. To check the correlation between radial velocity and $\mathrm{H} \alpha$ activity, we computed the linear Pearson correlation coefficients (IDL procedure correlate) between normalized $\mathrm{H} \alpha$ luminosity and differential radial velocity.

The results for each spectral order are shown in Fig. 9. Symbols are the same as in Fig. 7. Data for the first night are computed without the six spectra taken during the strong flare. In most of the spectral orders, the absolute value of the correlation coefficient is small $(<0.3)$ indicating no or very weak correlation between $\mathrm{H} \alpha$ emission and radial velocity. Thus, flaring shows no obvious effect on the radial velocities as long as $\Delta v$ is measured in spectral orders free of chromospheric emission lines. On the other hand, spectral orders containing prominent emission lines, namely orders $\# 70, \# 72$, and \#93, exhibit rather high (absolute) correlation coefficients. Here, activity strongly affects radial velocity measurements through chromospheric emission in the $\mathrm{Ca}, \mathrm{He}$, and $\mathrm{H} \alpha$ lines, although small flares cannot immediately be detected in the spectra themselves. These orders must to be omitted when searching for radial velocity variations in active $\mathrm{M}$ dwarfs.

In Fig. 9, we also show the correlation coefficients calculated from the entire first night, i.e., including the huge flare (plotted in red). As mentioned above, this flare substantially affected the whole spectrum including orders far away from $\mathrm{H} \alpha$ (see Fuhrmeister et al. 2008). The red crosses in Fig. 9 demonstrate that almost all spectral orders - including the ones containing mostly telluric lines - now show strong correlation with $\mathrm{H} \alpha$ emission. Thus, the radial velocity shift measured during this flare clearly is a systematic effect and not due to motions of the flare plasma. Spectra taken during flare events similar to this one therefore carry little information about the real radial velocity of the star (on the order of a few hundred $\mathrm{m} \mathrm{s}^{-1}$ ). Interestingly, the two orders containing the $\mathrm{H} \alpha$ line show an anti-correlation with $\mathrm{H} \alpha$ emission. An anti-correlation between radial velocity and $\mathrm{H} \alpha$ luminosity was also measured at higher accuracy in a much less active star by Kürster et al. (2003).

\section{Summary}

We have used UVES observations of the very active M dwarf $\mathrm{CN}$ Leo during three nights investigating the effect of flares on short-term radial velocity determinations. Radial velocities were measured relative to telluric absorption bands because no bracketing ThAr exposures or calibrations through an iodine cell were available. With this method, we could remove strong absolute shifts that occurred during the nights. Remaining intra-night drifts may be explained by pressure variations changing the dispersion of the spectrograph or by corotating spots on the surface of CN Leo. These smooth trends were neglected in our analysis.

During two nights, data were taken continuously for more than four hours. This allowed to investigate short-term variations of radial velocity. Many flaring events were observed during that time. The timescale of flares is less than an hour and several flares are covered by our data.

At the beginning of the first night, a huge flare occurred that dramatically changed the appearance of the whole spectrum. This flare had strong effect on the measured radial velocities in all spectral orders. Even spectral orders that predominantly contain telluric lines show radial velocity shifts on the order of a few hundred $\mathrm{m} \mathrm{s}^{-1}$. This dramatic kind of flaring events, however, is not a general problem for radial velocity surveys, because such an event is easily identified in the spectrum. In this case, the flare limits the radial velocity stability to $\sim 500 \mathrm{~m} \mathrm{~s}^{-1}$ in the peak spectrum, and to $\sim 50 \mathrm{~m} \mathrm{~s}^{-1}$ directly after the peak. Such events must be removed from the analysis.

Outside the huge flare, spectral orders containing prominent emission lines show large scatter. Radial velocities measured in the order containing the $\mathrm{H} \alpha$ line are anti-correlated to $\mathrm{H} \alpha$ emission strength. This was also reported in the very inactive M dwarf Gl 699 by Kürster et al. (2003).

In the orders free of prominent emission lines, the radial velocity jitter is below $10 \mathrm{~m} \mathrm{~s}^{-1}$. Outside the huge flare, CN Leo still showed flaring activity that enhanced the normalized $\mathrm{H} \alpha$ flux by $\lesssim 0.4$ dex. Despite this rather strong activity, no correlation was found between activity and radial velocity.

Even in one of the most active known M dwarfs, flares produced during average stellar activity have no effect on high precision radial velocities on the level of $10 \mathrm{~m} \mathrm{~s}^{-1}$ if one chooses 
spectral orders free from emission lines. This means that the influence of flares - the most obvious effect of stellar activity on the spectra of $\mathrm{M}$ dwarfs - is negligible in planet searches at least down to several $\mathrm{m} \mathrm{s}^{-1}$. Even in the presence of flaring, it may be possible to detect an earth-like planet inside the habitable zone of a late M dwarf.

Acknowledgements. I thank Andreas Seifahrt and Jacob Bean for many enlightening discussions on the subject, and Uwe Wolter and Carolin Liefke for providing the reduced data set. Research funding is acknowledged from the DFG under an Emmy Noether Fellowship (RE 1664/4-1). An encouraging and very helpful report by an anonymous referee is thankfully acknowledged.

\section{References}

Balthasar, H., Thiele, U., \& Wöhl, H. 1982, A\&A, 114, 357

von Bloh, W., Bounama, C., Cuntz, M., \& Franck, S. 2007, A\&A, 476, 1365
Bonfils, X., Mayor, M., Delfosse, X., et al. 2007, A\&A, 474, 293

Butler, R. P., Marcy, G. W., Williams, E., et al. 1996, PASP, 108, 500

Demory, B.-O., Gillon, M., Barman, T., et al. 2007, A\&A, 475, 1125

Desort, M., Lagrange, A.-M., Galland, F., Udry, S., \& Mayor, M. 2007, A\&A, 473, 983

Fuhrmeister, B., Liefke, C., Schmitt, J. H. M. M., \& Reiners, A. 2008, A\&A, 487, 293

Kaufer, A., D’Odorico, S., Kaper, L., \& Ledoux, C. 2007, UVES User Manual, ESO

Kürster, M., Endl, M., Rouesnel, F., et al. 2003, A\&A, 403, 1077

Lacy, C. H. 1977, ApJS, 34, 479

Pepe, F., Mayor, M., Galland, F., et al. 2002, A\&A, 388, 632

Reiners, A., \& Basri, G. 2007, ApJ, 656, 1121

Reiners, A., Homeier, D., Hauschildt, P. H., \& Allard, F. 2007, A\&A, 473, 245

Saar, S. H., \& Donahue, R. A. 1997, ApJ, 485, 319

Selsis, F., Kasting, J. F., Levrard, B., et al. 2007, A\&A, 476, 1373

Short, C. I., \& Doyle, J. G. 1998, A\&A, 336, 613

Tarter, J. C., Backus, P. R., Mancinelli, R. L., et al. 2007, Astrobiology, 7, 30

West, A. A., Hawley, S. L., Walkowicz, L. M., et al. 2004, AJ, 128, 426 